\title{
Maritime Mobile Channel Transmission Model Based on ITM
}

\author{
Yuwei Zhao \\ College of Information Science and Technology \\ Hainan University \\ Haikou, China \\ e-mail:315533591@qq.com
}

\author{
Jia Ren* \\ College of Information Science and Technology \\ Hainan University \\ Haikou, China \\ e-mail:wwwrenjia@gmail.com
}

\author{
Xun Chi \\ College of Information Science and Technology \\ Hainan University \\ Haikou, China \\ e-mail:chixun1989@vip.sina.com
}

\begin{abstract}
Aiming to characteristics of transmission path loss in mobile channel which is caused by complex electromagnetic environment on an open sea area, an improved Irregular Terrain Methodology (ITM) model is proposed in this paper. The algorithm first uses a Two-Path Model to calculate the transmission loss in $1 \mathbf{k m}$ 's distance on maritime; Secondly, the characteristics of radio wave propagation on an open sea was corrected by adding cloud, fog fading and rain loss to the mode; Finally, it compares the results obtained by the standard ITM model and the improved ITM model on Matlab simulation software. The results show that the improved ITM model can better reflect characteristics of pelagic mobile channel and can improve the quality of mobile channel transmission compared with standard ITM model.
\end{abstract}

Keywords-attenuation; path loss simulation;maritime mobile communication; radio wave propagation

\section{INTRODUCTION}

There is very big difference between maritime mobile channel and land mobile channel environments. On the maritime terrain, there are a few sea obstructions that have reduced the diffraction loss. Two facts of small grazing angle and lager a reflection of sea face have resulted very strong reflection effect in the microwave band, In addition, a complicated and changeable maritime climate, clouds, raindrops have seriously affected the maritime mobile channel transmission loss interference.

In order to explore the characteristics of maritime mobile channel transmission, literature [1] has predicted that the radio wave on maritime can be seen as a free space radio wave propagation; literature [2] has made a test of the maritime radio propagation characteristics in $1800 \mathrm{MHz}$ on the sea shore in Zhangzhou, Fujian. Literature [3] has proposed to use Okumura-Hata model and the Irregular Terrain Methodology (ITM) model to predict transmission loss of maritime mobile channel under the simulation test condition. Simulation test results have proven that Okumura-Hata model has some kinds of applicability under the following test conditions: distance of $1 \sim 100 \mathrm{~km}$, a frequency of $150 \sim 1920 \mathrm{MHz}$, and antenna height of $30 \sim 1000 \mathrm{~m}$. The simulation test has also found that the factors of sea surface refractivity, sea surface conductivity, dielectric constant, sea roughness climate types, and the location standards of antennas have affected on transmission loss of maritime mobile channel using ITM model working in band $20-40 \mathrm{GHz}$ and the path length of $1-2000 \mathrm{~km}$. Literature [4] has pointed out that the use of ITM model to predict path loss is more appropriate because Okumura-Hata model does not take into consideration specific sea face medium parameters. However, it did not take into consideration the following two aspects: (1) receiver ambient factors on the impact of electromagnetic transmission; (2) above two models without the ability to predict the field strength in path length of less than $1 \mathrm{~km}$. To meet the need of simulation modeling of marine Communication, it is necessary to amend and supplement of ITM model.

Focusing on solving two problems mentioned above, this paper improves and amends ITM model by using the dual-path model, clouds fading model, and rain attenuation model for establishing the loss in value of the wave propagation prediction in maritime and for fitting in with the needs of the building of simulation modeling of marine Communication.

\section{CORRECTING ITM MODEL}

\section{A. Introduction to ITM model}

Irregular terrain model (ITM) is called irregular terrain model, which predicts long-term median transmission loss over irregular terrain relative to free-space transmission loss. It is also called semi-empirical prediction models. The median transmission loss model is defined the distribution function of the distance [5], as follows: 


$$
\left\{\begin{array}{cl}
A_{e}+k_{1} d+k_{2} \log _{10} d, & 1 \leq d \leq d_{L S}= \\
A_{e d}+m_{d} d, & d_{L S} \leq d \leq d_{x} \\
A_{e s}+m_{s} d, & d_{x} \leq d
\end{array}\right.
$$

The terrain topography and tropospheric diffraction geometry was taken accounted in the median transmission loss model to predict the value of median transmission attenuation, shown in equation (1). Attenuation relative to free space is calculated using two-ray optics formulas in the line of sight $\left(1 \leq \mathrm{d} \leq \mathrm{d}_{\mathrm{LS}}\right)$ while discrimination was used to evaluate attenuation beyond line of sight $\left(\mathrm{d}_{\mathrm{LS}} \leq \mathrm{d} \leq \mathrm{d}_{\mathrm{X}}\right)$. $A$ weighted average of estimates of diffraction attenuation over a double knife edge and over irregular terrain was computed [6]. Forward scatter attenuation was use the longer distances $\left(\mathrm{d}_{\mathrm{X}} \leq \mathrm{d}\right)$ [7].

\section{B. Correcting ITM}

Dual-path propagation prediction model, Rain Attenuation model and cloud loss model were integrated to ITM model to perform the prediction of median transmission loss for maritime communication,

1) Dual-path propagation prediction model. Free space propagation model is an ideal propagation model, which exists in a uniform medium with isotropic, no absorption, and zero conductivity. It is clear that the model could not been used alone in the maritime mobile channel. There is a strong direct signal and a reflected wave from sea surface in the sea within $1 \mathrm{~km}$. The shorter distance can be seen as planar transmission. This paper proposes a dual-path propagation model which not only take the direct path into consideration, but also the between transmitter and receiver sea reflection path into consideration within $1 \mathrm{~km}$ offshore. Research shown that predicting large-scale signal strength is very accurate [8], shown in Fig.1:

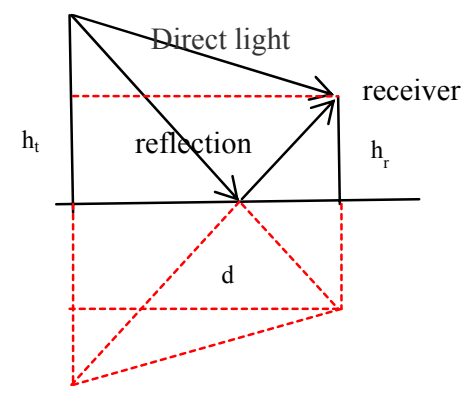

Fig. 1. Schematic double-pass reflection prediction model

Horizon and ground reflection path difference is defined as follows:

$$
\begin{gathered}
\Delta=r_{2}-r_{1}=\sqrt{\left(h_{t}+h_{r}\right)^{2}+d^{2}}- \\
\sqrt{\left(h_{t}-h_{r}\right)^{2}+d^{2}}
\end{gathered}
$$

$\mathrm{r}_{1}, \mathrm{r}_{2}$ is the distance between the sending and receiving end and the point of reflection respectively, $h_{t}, h_{r}$ is the antenna height of sending and receiving end respectively, $\mathrm{d}$ is the distance between the receiver and sender.

Therefore, the phase difference between two electric field components can be expressed as:

$$
\theta_{\Delta}=\frac{2 \pi \Delta}{\lambda}=\frac{4 \pi h_{t} h_{r}}{\lambda d}
$$

Where $\theta_{\Delta}$ represents the phase difference, $\lambda$ represents wavelength. The square of receive field strength and free space field strength is:

$$
\begin{gathered}
\left|\frac{E_{r e c}}{E_{f s}}\right|^{2} \approx 2\left(1-\cos \theta_{\Delta}\right)=4\left[\sin \left(\frac{\theta_{\Delta}}{2}\right)\right]^{2}= \\
4\left[\sin \left(\frac{2 \pi h_{t} h_{r}}{\lambda d}\right)\right]^{2}
\end{gathered}
$$

Formula (4) provides accurate received electric field intensity to the two-path model. $\mathrm{E}_{\text {rec }}$ is the received field strength, $\mathrm{E}_{\mathrm{fs}}$ is a free-space field strength values.

When $d$ is much larger than the antenna height, the formula (4) can simplify as:

$$
\left|\frac{E_{r e c}}{E_{f s}}\right|^{2} \approx\left(\frac{4 \pi h_{t} h_{r}}{\lambda d}\right)^{2}
$$

Therefore, the path gain is equal to formula (4) multiplied by the free space loss formula:

$$
\begin{gathered}
\frac{P_{r}}{P_{t}}=4\left[\sin \left(\frac{2 \pi h_{t} h_{r}}{\lambda d}\right)\right]^{2} \times\left(\frac{\lambda}{4 \pi d}\right)^{2} \approx \\
\left(\frac{4 \pi h_{t} h_{r}}{\lambda d}\right)^{2} \times\left(\frac{\lambda}{4 \pi d}\right)^{2}=\left(\frac{h_{t} h_{r}}{d^{2}}\right)^{2}
\end{gathered}
$$

Equation (6) shows, when the distance $d$ is large, received power increasing with the distance was fourth power, which is faster than the loss of the free space, $\frac{P_{r}}{P_{t}}$ means the path gain. In terms of units of $\mathrm{dB}$, the path loss is expressed as:

$$
\begin{gathered}
P_{L}(d B)=10 \log \left(\frac{d^{2}}{h_{t} h_{r}}\right)^{2}= \\
20 \log \left(\frac{d^{2}}{h_{t} h_{r}}\right)
\end{gathered}
$$
(6).

Where, $P_{L}(\mathrm{~dB})$ is the representation of $\mathrm{dB}$ for formula

2) Clouds Attenuation. Fog is composed of water droplets suspended in the air near the ground and has strong effect on the low-frequency sound waves and subsound attenuation. Cloud is composed of tiny water droplets, water droplets' diameter in $0.001-0.4 \mathrm{~mm}$. Since the size of droplet clouds is smaller than the wavelength of microwave and millimeter, generally, cloud can be approximated using the Rayleigh for droplet clouds extinction cross section. Since the absorption cross section is much larger than cloud droplets scattering cross-section, the body is approximately equal to the body extinction coefficient absorption coefficient, the value of all clouds particles per unit volume equals to the 
absorption cross section, the specific attenuation within a cloud or fog can be expressed as:

$$
r_{c}=K_{I} M
$$

Where $K_{I}$ is specific attenuation coefficient $(\mathrm{dB} / \mathrm{km}) /\left(\mathrm{g} / \mathrm{m}^{3}\right) ; \mathrm{M}$ is liquid water density in the cloud or fog $(\mathrm{g} / \mathrm{m} 3)$.

$\mathrm{M}$ is calculated by theorem of cloud droplet size distribution :

$$
M=\frac{4 \pi}{3} \rho_{w} \int_{0}^{\infty} a^{2} n(a) d a
$$

Where $\rho_{\mathrm{W}}$ is water density in the cloud or fog $\left(\mathrm{g} / \mathrm{m}^{3}\right)$, a is cloud droplet radius ( $\mathrm{mm}), \mathrm{n}(\mathrm{a})$ is cloud droplet size distribution density function.

Typical cloud liquid water content is $0.1 \sim 1(\mathrm{~g} / \mathrm{m} 3)$, when rough estimation, $\mathrm{M}=0.15\left(\mathrm{~g} / \mathrm{m}^{3}\right)$ for rain cloud; for fog that $M=0.05\left(\mathrm{~g} / \mathrm{m}^{3}\right.$ ) (medium fog, visibility of the order of $300 \mathrm{~m}$ or $\mathrm{M}=0.5\left(\mathrm{~g} / \mathrm{m}^{3}\right)$ (thick fog, visibility of the order of $50 \mathrm{~m}$ ).

The literature [10] shows that a mathematical model based on Rayleigh scattering, which uses a double-Debye model for the dielectric permittivity of water, can be used to calculate the value of $\mathrm{K}_{1}$ for frequencies up to 1000 GHz:

$$
\begin{gathered}
K_{1}=\frac{0 \cdot 819 f}{\varepsilon "\left(1+\eta^{2}\right)} \\
\qquad \eta=\frac{2+\varepsilon^{\prime}}{\varepsilon^{\prime \prime}}
\end{gathered}
$$
by:

The complex dielectric permittivity of water is given

$$
\begin{gathered}
\varepsilon^{\prime \prime}(f)=\frac{f\left(\varepsilon_{0}-\varepsilon_{1}\right)}{f_{p}\left[1+\left(f / f_{p}\right)^{2}\right]}+\frac{f\left(\varepsilon_{1}-\varepsilon_{2}\right)}{f_{s}\left[1+\left(f / f_{s}\right)^{2}\right]} \\
\varepsilon^{\prime}(f)=\frac{\varepsilon_{0}-\varepsilon_{1}}{1+\left(f / f_{p}\right)^{2}}+\frac{\varepsilon_{1}-\varepsilon_{2}}{1+\left(f / f_{s}\right)^{2}}+\varepsilon_{2}
\end{gathered}
$$

$\varepsilon^{\prime} 、 \varepsilon^{\prime \prime}$ represents a real part and imaginary part of the complex permittivity of liquid. It can be seen in the Rayleigh approximation. Cloud attenuation has nothing to do with spectral distribution drops, which is only moisture related. Furthermore, the complex permittivity of the water is a function of temperature. Temperature has a great influence on cloud attenuation.

From the above equations, we can analyze the clouds decay rates at different frequencies temperatures, where temperature is $-5^{\circ} \mathrm{C}, 0{ }^{\circ} \mathrm{C}, 20^{\circ} \mathrm{C}, 30^{\circ} \mathrm{C}$, respectively. As can be seen from following figure, propagation at the same frequency, clouds decay rate increases as the temperature decreases; propagation at the same temperature, the cloud decay rate increases with the wave frequency.



Fig. 2. Clouds at different temperatures vs. frequency attenuation coefficient

3) Rain Attenuation. In marine environment, rainfall is generally very large. An electromagnetic wave into a rain layer will attenuate. This is called as a rain fade. Rain fades include the fade caused by raindrops absorption and the fade caused by raindrops scattering. The degree of rain attenuation has a comparable relationship with the ratio of raindrop diameter and wavelength. Research has shown that for frequencies above $1 \mathrm{GHz}$, rain attenuation is an important factor affecting its spread [11] [12] [13].

High Power Microwave (HPM) model which is applied to radio waves at frequencies below $350 \mathrm{GHz}$ can be used to estimate rain attenuation [14]. In the path length for the r0, the relationship between rain attenuation $A_{R}$ and propagation path rain attenuation rate $\gamma_{R}$ $(\mathrm{dB} / \mathrm{km})$ is defined as following:

$$
A_{R}=\int_{0}^{r 0} \gamma_{R} d r, \gamma_{R}=k R^{\alpha}
$$

Let set $\mathrm{R}$ is the $\operatorname{rainfall}$ intensity $(\mathrm{mm} / \mathrm{h}), \mathrm{k}, \alpha$ is related parameters of wave frequency $f(\mathrm{GHz})$ respectively, $\theta$ is path elevation is polarization angle, $\mathrm{K}$ and $\alpha$ can be expressed as the following formula:

$$
\begin{gathered}
k=\left[k_{H}+k_{V}+\left(k_{H}-k_{V}\right) \cos ^{2} \theta \cos 2 \tau\right] / 2 \\
\alpha=\frac{\left[k_{H} \alpha_{H}+k_{V} \alpha_{V}+\left(k_{H} \alpha_{H}-k_{V} \alpha_{V}\right)(\cos \theta)^{2} \cos 2 \tau\right]}{2 k}
\end{gathered}
$$

Let set $\mathrm{H}, \mathrm{V}$ to denote the value of response parameters in horizontal polarization and vertical polarization, $\theta$ is path elevation, $\tau$ is polarization angle (Horizontal polarization is $0^{\circ}$; vertical polarization is $90^{\circ}$; circular polarization is $45^{\circ}$ ).

The formula of $k_{H} 、 k_{V} 、 \alpha_{H} 、 \alpha_{V}$ are expressed as follows :




$\alpha_{v}=\left\{\begin{array}{l}\frac{(1.0246 \ln f-1.9462)^{2}+0.9048}{(1.1141 \mathrm{n} f-2.0940)^{2}+0.7181}, 1 \leq f \leq 20 \mathrm{GHz} \\ 0.6833+\frac{0.4494}{1+1.8700 * 10^{-4} f^{2.2803}}, 20 \leq f \leq 400 \mathrm{GHz}\end{array}\right.$

The curve of $k_{H}, k_{V} 、 \alpha_{H}, \alpha_{V}$ and frequency $(1 \leq \mathrm{f} \leq 20 \mathrm{GHz})$ can be obtained from the above equations, as shown in Fig.3:

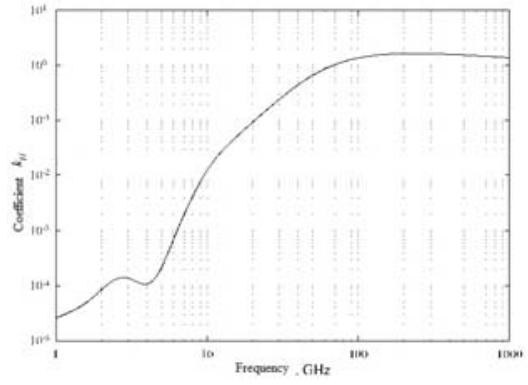

(a) $k_{H}$ and frequency in curve

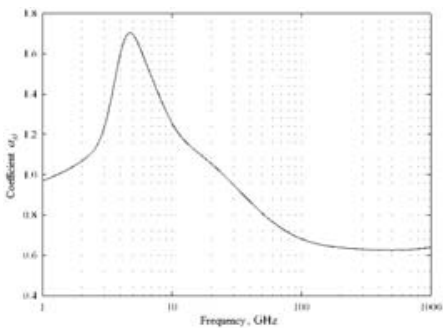

(b) $k_{V}$ and frequency in curve

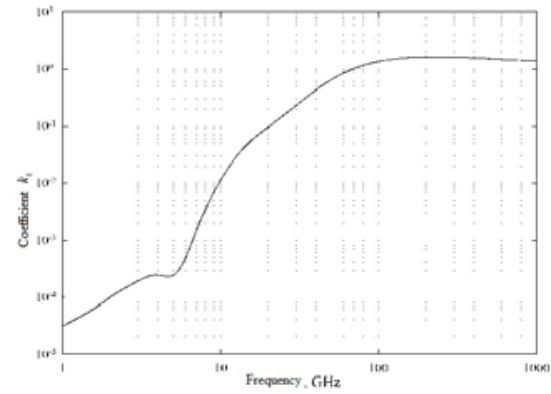

(c) $\alpha_{H}$ and frequency in curve



(d) $\alpha_{V}$ and frequency in curve

Fig. 3. $k_{H}, k_{V}, \alpha_{H}, \alpha_{V}$ and frequency in curve
Let path elevation angle $\theta=20^{\circ}, \quad \tau=0^{\circ} \quad$ (Horizontal polarization ) , frequency $1 \leq \mathrm{f} \leq 20 \mathrm{GHz}$, Under these conditions the curve of rain attenuation rate $\gamma_{R}$ and radio Frequency $\mathrm{f} / \mathrm{GHz}$ can be derived.

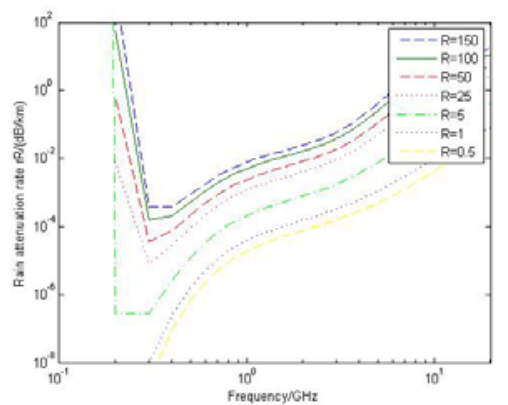

Fig. 4. rain attenuation rate $\gamma_{R}$ and radio Frequency $\mathrm{f} / \mathrm{GHz}$ in curve

\section{CALCULATION OF TRANSMISSION FADING}

Reference value of transmission loss $\mathrm{L}_{\mathrm{cr}}[7]$ is the sum of free space fading two-path transmission $\mathrm{P}_{\mathrm{L}}$, reference loss of free space $A_{c r}$, clouds fading $A$, and rain attenuation ar: That is:

$$
\mathrm{L}_{\mathrm{cr}}=\mathrm{P}_{\mathrm{L}}+\mathrm{A}_{\mathrm{cr}}+\mathrm{A}+\mathrm{ar} \mathrm{dB}
$$

\section{Simulation}

\section{A. ITM channel fading simulation analysis}

ITM model uses the frequency ranges from 20 to $40000 \mathrm{MHz}$ generally, transceiver antenna height from 0.5 to $3000 \mathrm{~m}$, covering a radius from 1 to $2000 \mathrm{~km}$, surface index from 250 to $400 \mathrm{Ns}[7]$.

According to the ITM model established in 3.1 , the measured data from literature [2] is used to increase the credibility of the algorithm. Literature [4] has tested signal fading in the East China Sea in Zhangzhou, Fujian. The base height is $200 \mathrm{~m}$, frequency is $1800 \mathrm{MHz}$, the mobile station height is $10 \mathrm{~m}$, and the communication distance is 50 to $90 \mathrm{~km}, 1 \mathrm{~km}$ test interval of a point. The test in literature [2] was taken in the actual environment its main parameters of the simulation environment are as follows:

(1) Frequency: $1800 \mathrm{MHz}$;

(2) Transmitting antenna height: 200m;

(3) Receiving antenna height: $10 \mathrm{~m}$;

(4) Climate type: Subtropical maritime climate;

(5) Sea surface roughness: 1;

(6) Antenna standard location's impact on fading: Random;

(7) Sea dielectric constant: 81 ;

(8) Sea surface conductivity: 5;

(9) Sea refractive index:320Ns;

(10) Antenna polarization: Horizontal polarization;

Transmission loss prediction model is shown in Fig.5: 




Fig. 5. Prediction of ITM model channel fading

As shown in Fig.5, when predicting the transmission loss at sea, simulation results using the ITM model appears an inflection point at about $65 \mathrm{~km}$, the slope becomes larger and the curve becomes steep. This is the reason the curvature of the earth line of sight at this position collapsed, generating the transition point Diffraction.

\section{B. Improved ITM channel fading simulation analysis}

The applicable range of ITM model is 1 to $2000 \mathrm{~km}$, but this model does not provide correction factors on the environment near the receiver, and not consider multipath propagation. In this paper ITM model is improved by adding Two-Path Model to calculate the transmission loss in $1 \mathrm{~km}$ 's distance on maritime. In addition, cloud, fog fading and rain loss are considered.

Using Matlab simulation platform for simulation analysis, the results have shown as following:

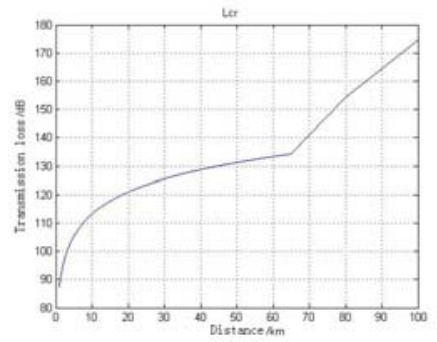

Fig. 6. Channel model transmission loss

The computer simulation environment established from the actual environment in literature[2], parameters of measured data, and simulation data in shown in 5.1 were used to predict transmission loss. Matlab simulation platform was used to simulate transmission loss. The comparison chart of improved model and standard ITM model is shown as follow

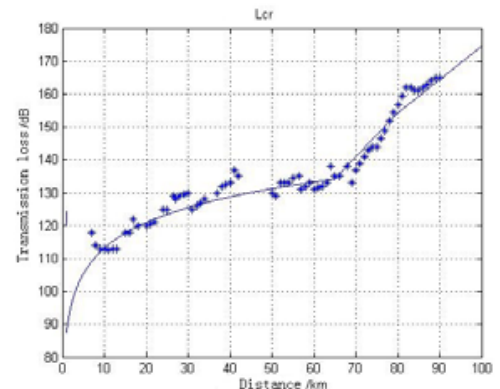

Fig. 7. Comparison of predicted data and measured data

Fig.7 shows the improved ITM loss prediction model is consistent with the measured data, simulation curve consistent with the measured curve after smoothing out several outliers test data, inflection point appears at $65 \mathrm{~km}$ both in prediction model and measured data. Compared with ITM model prediction, the improved ITM model has larger measuring range, is more universal and more close to the measured environment.

\section{SUMMARY}

In this paper, characteristics of transmission path loss in maritime mobile channel are analyzed, an improved Irregular Terrain Methodology (ITM) model is proposed. The algorithm first uses a Two-Path Model to predict the transmission loss in $1 \mathrm{~km}$ 's distance on maritime. Then considering the environmental impact of weather factors, such as cloud, fog fading, rain loss is added to evaluation. Compared results obtained from the simulation results and the theoretical analysis, we have found that the cloud attenuation is more obvious at frequencies above $10 \mathrm{GHz}$ for microwave communications and can be used as an important factor in the decline of maritime communications. The results show that the improved ITM model conforms to the measured data environment better, which improve the accuracy of predicted maritime mobile channel path loss. Therefore, the authors believe that improved ITM model can be adopted to predict maritime channel transmission loss, can make forecasts more realistic.

\section{ACKNOWLEDGEMENTS}

This was supported by National Natural Science Foundation of China(61162010), partly by The Research Fund for the Master Program of Higher Education of Hainan Province of China.

\section{REFERENCES}

[1] Wang xiang-min. A Study on the Atmospheric Duct over Ocean and Its Prediction [D]. Nanjing University of Information Science\&Technology.2007.

[2] He Qun, Huang yun-peng. Discussion on Radio Propagation Model over Sea Level [J]. Designing Techniques of Posts and Telecommunications.2004, 2:011.

[3] Mo H, Chen B, Shen C. Radio propagation prediction model for maritime mobile communication [J]. 2012. 
[4] Phillips C, Sicker D, Grunwald D. The Stability of the LongleyRice Irregular Terrain Model for Typical Problems [J]. arXiv preprint arXiv: 1109.1843, 2011.

[5] Tse D, Viswanath P. Fundamentals of wireless communication [M] Cambridge university press, 2005.

[6] Chai Wei-chen, Mao Jing-li. A Practical Location Scheme Based On GSM [J]. Computer \&Information Technology, 2010, 17(1):14.

[7] Longley A G, Rice P L. Prediction of tropospheric radio transmission loss over irregular terrain. A computer method1968[R]. INSTITUTE FOR TELECOMMUNICATION SCIENCES BOULDER CO, 1968.

[8] Li si-jia, Mao yu-quan, Yao Xu. Adaptive Markov model for channel path fading of near space UAV [J]. Application Research of Computers, 2010, 27(12).

[9] Mao X, Liu Y L, Chen L J, et al. A More Precise Empirical Formula for Estimating Normalized Fog Attenuation in the Millimeter-Wave Frequency Range 30 $100 \mathrm{GHz}$ [J]. Journal of Infrared, Millimeter, and Terahertz Waves, 2013: 1-8.
[10] Recommendation I. 840: Attenuation due to clouds and fog [J]. ITU-R Recommendations, P Series Fasicle, ITU, Geneva, Switzerland, 1999.

[11] Shifeng K, Hongguang W. Analysis of microwave over-thehorizon Propagation on the sea[C]//Microwave Conference, 2009. APMC 2009. Asia Pacific. IEEE, 2009: 1545-1548.

[12] Gurung S, Zhao J. Attenuation of Microwave Signal and Its Impacts on Communication System [J]. Graduate Project, Department of Electrical Engineering, College of Engineering, University of North Texas, 2011.

[13] Zilinskas M, Tamosiunaite M, Tamosiunas S, et al. The influence of the climatic peculiarities on the electromagnetic waves attenuation in the Baltic Sea region[J]. PIERS Online, 2008, 4(3): 321-325.

[14] Recommendation I. 838-3. Specific attenuation model for rain for use in prediction methods $[\mathrm{J}]$. International Telecommunication Union, Geneva, 2005. 